\title{
Composición, origen y valor de conservación de la Ictiofauna del Río San Pedro (Cuenca del Río Valdivia, Chile)
}

\section{Composition, origin and conservation value of the San Pedro River Ichthyofauna (Valdivia River Basin, Chile)}

\author{
Evelyn Habit ${ }^{1}$, Pedro Victoriano ${ }^{2}$ \\ ${ }^{1}$ Unidad de Sistemas Acuáticos, Centro de Ciencias Ambientales EULA-Chile, Universidad de Concepción. Concepción, Chile. Casilla \\ $160-$ C. \\ ${ }^{2}$ Facultad de Ciencias Naturales y Oceanográficas. Departamento de Zoología, Universidad de Concepción. Concepción, Chile. \\ Casilla160-C. \\ E-mail: ehabit@udec.cl
}

\begin{abstract}
RESUMEN
Basado en muestreos de tres años consecutivos y revisión de literatura, describimos la composición, singularidad y estado de conservación de la ictiofauna del río San Pedro, en la cuenca del río Valdivia. Además, analizamos los posibles orígenes de esta ictiofauna, postulando que parte de la cuenca del río Valdivia ha funcionado como un sumidero, principalmente su parte alta, con aportes de fuentes tanto costero-Pacíficas como del Este de Los Andes. Concluimos que el río San Pedro es de particular valor ictiofaunístico por su alta riqueza específica, endemismos y características biogeográficas.

Palabras clave: Fauna íctica, Río San Pedro, Endemismos, Conservación, Chile.

ABSTRACT

Based on three consecutive years of sampling and bibliographic revision, the composition, singularity and conservation status of the San Pedro River ichthyofauna is described. In addition, the potential origin of this Valdivia River ichthyofauna was analyzed and this basin is proposed to have worked like a sink, mainly the higher zone, having been colonized both from Coastal-Pacific as from Eastern Andes sources. It is concluded that this river has a particular conservation value due to its high species richness, endemism and biogeographic characteristics.
\end{abstract}

KEYwords: Fish fauna, San Pedro River, endemisms, conservation, Chile.

\section{INTRODUCCIÓN}

La ictiofauna chilena se caracteriza por estar constituida por una riqueza moderada en relación a otras áreas de Sudamérica, y por un alto grado de endemismo (Vila et al. 1999; Dyer 2000; Habit et al. 2006a). Este patrón puede estar asociado a dos aspectos causales. El primero se relaciona con el hecho que los regímenes ambientales que han imperado en los sistemas dulceacuícolas chilenos han limitado su enriquecimiento ictiofaunístico a lo largo de su historia. Entre tales características, cuenta la oligotrofía de los ecosistemas fluviales chilenos y la alta rigurosidad, en términos de elevada velocidad de corriente, la ocurrencia de rangos térmicos bajos, y el haber estado sometidos a condiciones de cambios climáticos extremos (glaciaciones, vulcanismo y movimientos tectónicos, entre otros procesos históricos)
(McCulloch et al. 2005, Hulton et al. 2002). El segundo aspecto, el alto endemismo, está relacionado directamente con el nivel de aislamiento que presentan las cuencas chilenas respecto a las demás dentro de Sudamérica. En este sentido, la presencia del desierto por el norte, la cordillera por el este, y el rigor de los cambios paleoclimáticos fríos por el sur, han constituido escenarios que albergan una diversidad generada al margen de sus sistemas vecinos, tales como la Patagonia Argentina o los sistemas al norte del Desierto de Atacama, que forman parte de una provincia ictiofaunística particular (Dyer 2000). De hecho, y no sólo para la ictiofauna, la mayor concentración de riqueza de especies se presenta en la zona central de Chile, lo cual le ha conferido la calidad hot spot de biodiversidad (Myers 2003). Esto implica a su vez la presencia de altos niveles de complejidad biológica en los diferentes niveles que conforman el concepto de biodiversidad. Tal 
concentración en el centro de Chile se explica, por una parte, por la mantención histórica de sistemas ecológicos antiguos que preceden al levantamiento andino, y por otra, a una mayor estabilidad y menor rigurosidad climática. Esta situación decae hacia el sur de Chile, a medida que nos acercamos a la zona de los bosques templados y subantárticos donde, en general, la biota se empobrece y constituye comunidades notoriamente más simples (Markgraf 1989). Sin embargo, la menor riqueza de especies no implica un menor valor de su ictiofauna, principalmente porque los procesos históricos a los que han estado sometidos los ecosistemas australes chilenos, dentro de los cuales se encuentra la cuenca del río San Pedro, corresponden a procesos únicos en el planeta. Estos procesos han sido solo equivalentes en otros continentes australes (Oceanía), y en el hemisferio norte, pero para una fauna completamente diferente (Benn \& Evans 2010).

Los patrones de distribución latitudinal de los peces de agua dulce de Chile están altamente correlacionados con factores históricos, entre los que destacan eventos geológicos complejos y reiterados, como los ciclos glaciales. A esto se suma el efecto de la diagonal árida que resulta de la combinación del surgimiento de Los Andes y de su interrelación con las corrientes aéreas predominantes. De esta manera, a lo largo del gradiente latitudinal en Chile, diversos grupos como los macroinvertebrados bentónicos (Valdovinos 2006) y organismos terrestres (Villagrán \& Armesto 2005), presentan su mayor número de especies en la zona Centro-Sur del país $\left(36^{\circ} \mathrm{S}-41^{\circ} \mathrm{S}\right)$. Esto también ocurre con los peces de agua dulce, los cuales presentan un claro patrón de incremento en el número de especies (presentes) desde los extremos norte y sur hacia la zona centro-sur (Vila et al. 1999). Dyer (2000) definió tres provincias biogeográficas en Chile, de acuerdo a su fauna de peces de agua dulce, i.e., Provincia de Atacama, Chilena y Patagónica. De éstas, la Provincia Chilena es la que contiene mayor diversidad, de manera que las cuencas hidrográficas con mayor número de especies nativas son aquellas ubicadas entre los ríos Itata y Bueno, tramo en el que se ubica la del río Valdivia. Según lo descrito previamente, cada una de ellas albergaría 16 especies nativas, a excepción de la cuenca del río Biobío, la cual contiene 17 especies, el mayor número de especies de peces en una cuenca en Chile (Vila et al. 1999). Es decir, entre las cuencas más ricas en peces nativos de Chile está la cuenca del Río Valdivia, de la cual es parte el río San Pedro. Considerando estos antecedentes generales, efectuamos aquí un análisis de la composición y singularidad de la ictiofauna presente en el sistema fluvial del río San Pedro, haciendo alcances sobre sus orígenes.

\section{MATERIALES Y MÉTODOS}

Para describir la composición y analizar el valor y singularidad de la fauna de peces del río San Pedro, se llevaron a cabo muestreos intensivos en el área y una revisión de literatura. Los muestreos en el río San Pedro abarcaron desde su nacimiento en el desagüe del Lago Riñihue hasta su confluencia con el río Quinchilca, donde se establecieron 35 sitios de muestreo (Figura 1). Estos sitios fueron muestreados en promedio tres veces al mes, entre octubre de 2005 y febrero de 2007 y entre junio de 2007 y febrero de 2008. Las artes de pesca utilizados correspondieron a pesca eléctrica, redes agalleras monofilamento y redes de arrastre. La pesca se efectuó siempre con devolución, por lo cual, las redes agalleras se revisaron cada media hora en el curso de los días de muestreo en cada sitio.

\section{RESULTADOS Y DISCUSIÓN}

La fauna de peces nativos del río San Pedro está compuesta por 14 especies (Tabla 1). Es decir, el 81\% de las especies nativas registradas en la cuenca del río Valdivia, incluyendo lagos, tributarios y ríos principales (Campos 1985, Vila et al. 1999, Ruiz y Marchant 2004), habitan en el río San Pedro, el cual es un tramo proporcionalmente corto respecto del total de la cuenca ( $\mathrm{ca} 40$. $\mathrm{km}$ de largo, es decir, menos del 40\%). Las especies citadas para la cuenca del río Valdivia pero que no se encuentran en el río San Pedro corresponden a la lamprea chilena (Mordacia lapicida Gray 1851), el pejerrey estuarino (Odontesthes brevianalis (Günther 1880)) y la pocha de Valdivia (Cheirodon kiliani Campos 1982). Estas tres especies se asocian típicamente a las partes bajas de los ríos, por lo que su ausencia en el río San Pedro es esperable. Además, la especie Ch. kiliani está descrita como una especie "rara" o poco abundante, que se encontraría restringida a pozas cerca de la ciudad de Valdivia y sectores costeros hacia Lanalhue (Ruiz \& Marchant 2004).

Generalmente, en Chile los ríos correntosos o ritrales albergan de tres a cinco especies nativas (Habit et al. 2006b). Por ello, dado que el río San Pedro es relativamente corto y de marcadas características ritrales, su riqueza de especies nativas igual a 14, es muy alta. Ello lo constituye en un sistema fluvial singular desde el punto de vista de su biodiversidad íctica. Otra singularidad de este sistema es la presencia de Diplomystes camposensis Arratia 1987 (tollo o bagre de agua dulce), siluriforme microendémico exclusivo de la cuenca del río Valdivia. La cuenca hidrográfica de este río también representa el extremo norte de la distribución de dos especies principalmente patagónicas: Galaxias platei Steindachner 1898 y Hatcheria macraei (Girard 1855). En tercer lugar, el río San Pedro es una de las cuencas con mayor riqueza y abundancia de galáxidos en Chile, donde habitan las dos especies del género Galaxias, dos de Aplochiton y una de Brachygalaxias (Habit et al. 2010). Por último, y a nivel de la cuenca completa del río Valdivia, si la especie 
Aplochiton marinus (Eigenmann, 1927), fuera una buena especie (ya que es dudosa según Dyer 2000), la cuenca del río Valdivia albergaría un total de seis especies de galáxidos, y un total de 18 especies nativas.

Características y Distribución de especies nativas EN EL Río SAN Pedro

Geotria australis Gray, 1851 ("lamprea de bolsa"; Figura 2a, Tabla 1)

Este ciclóstomo es una especie anádroma obligatoria. Los juveniles se desarrollan en aguas dulces de fondos blandos de esteros, ríos y lagos (Campos et al. 1993; Ruiz 1993; Jellyman \& Glova 2002) y los adultos habitan en el mar. La migración de adultos a aguas dulces ocurre una vez al año, recorriendo distancias de hasta $12,6 \mathrm{~km}$ al día (Jellyman et al. 2002). La migración es estimulada por el incremento del caudal y la mayor parte de los adultos busca tributarios donde desovar (Jellyman et al. 2002). Los juveniles que se desarrollan en fondos blandos, emprenden una migración en dirección al mar cuando han alcanzado el estado de larva macroftalmia. Es una especie de muy amplia distribución mundial, estando presente en Nueva Zelandia, Australia y Sudamérica. En el río San Pedro se la encuentra desde el desagüe del lago Riñihue hasta el río Calle-Calle.
Diplomystes camposensis Arratia, 1987 ("tollo"; Figura 2b, Tabla 1)

Los peces de la Familia Diplomystidae, se consideran como basales o grupo hermano primitivo de todos los demás bagres o peces gato (Grande 1987; Arratia 1992; dePinna 1998; Sullivan et al. 2006). Son peces que retienen caracteres primitivos, tales como la existencia de un hueso maxilar bien desarrollado con dientes, carácter sólo descrito para bagres extintos (Lundberg et al. 2004). Diplomystes camposensis es microendémico de la cuenca del Valdivia, aunque existe cierta controversia al respecto. $\mathrm{Su}$ distribución geográfica ha sido citada por Campos et al. (1998) y Ruiz \& Marchant (2004) para las Regiones IX y X, lo cual podría abarcar desde el río Imperial a la Isla de Chiloé. Arratia (1987) y Dyer (2000) indican que es una especie presente sólo en la cuenca del río Valdivia. GESAM, en la propuesta de clasificación de categorías de conservación de CONAMA (2008), indica que estaría presente en los ríos Imperial y Valdivia. De acuerdo a nuestros estudios, D. camposensis es endémica de la cuenca del río Valdivia. En el río San Pedro tiene una distribución continua desde el desagüe del lago Riñihue hasta el río Calle-Calle.

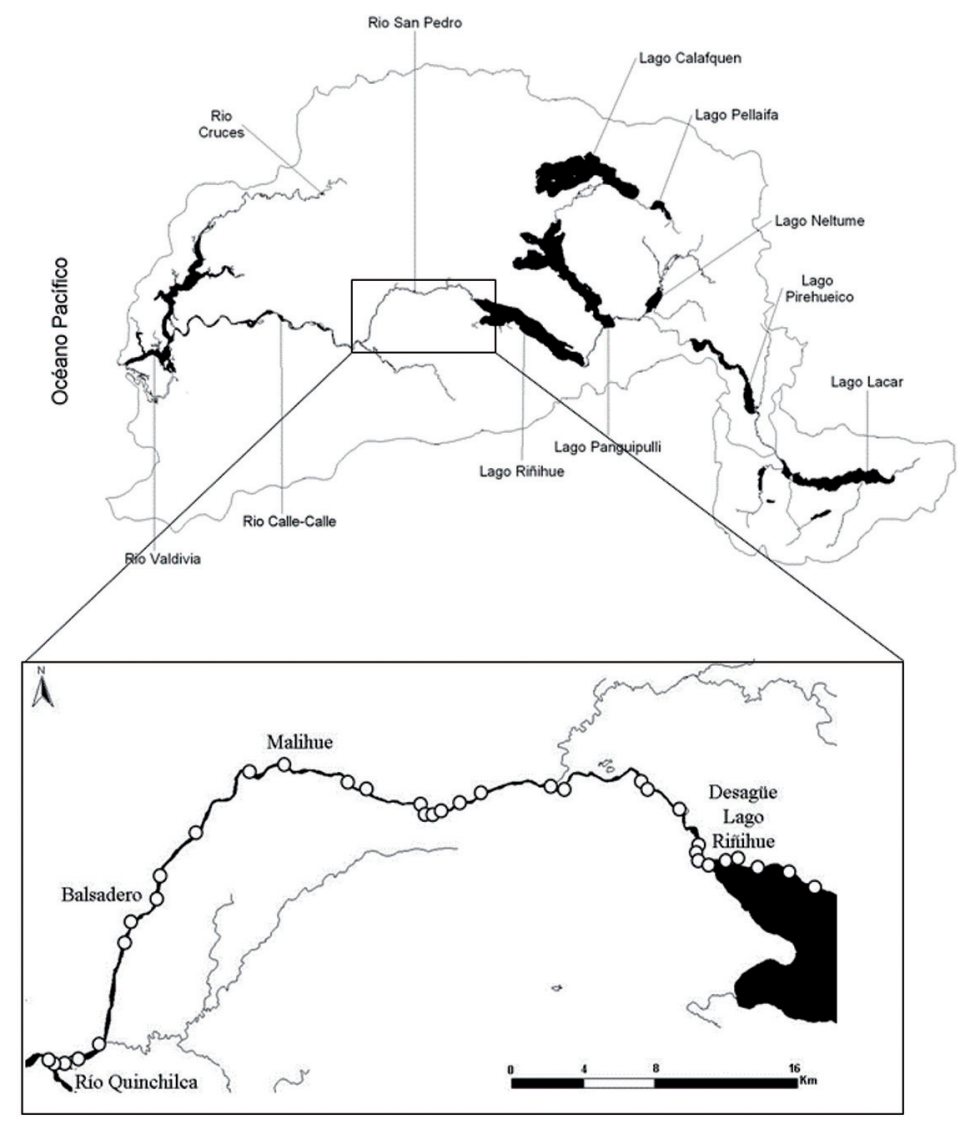

Figura 1. Cuenca del río Valdivia y ampliación de la zona de estudio en el río San Pedro. Los círculos blancos indican los sitios de muestreo.

Figure 1. Valdivia River watershed and detail of the study area in the San Pedro River. White circles correspond to sampling sites. 
Tabla 1. Ictiofauna nativa descrita para la cuenca del río Valdivia. Se indica su distribución en Chile. Su distribución dentro del río san Pedro se describe en el texto.

Table 1. Native fish fauna described for the Valdivia River basin. Species distributions along Chile are in the table. Species distribución along the San Pedro river.

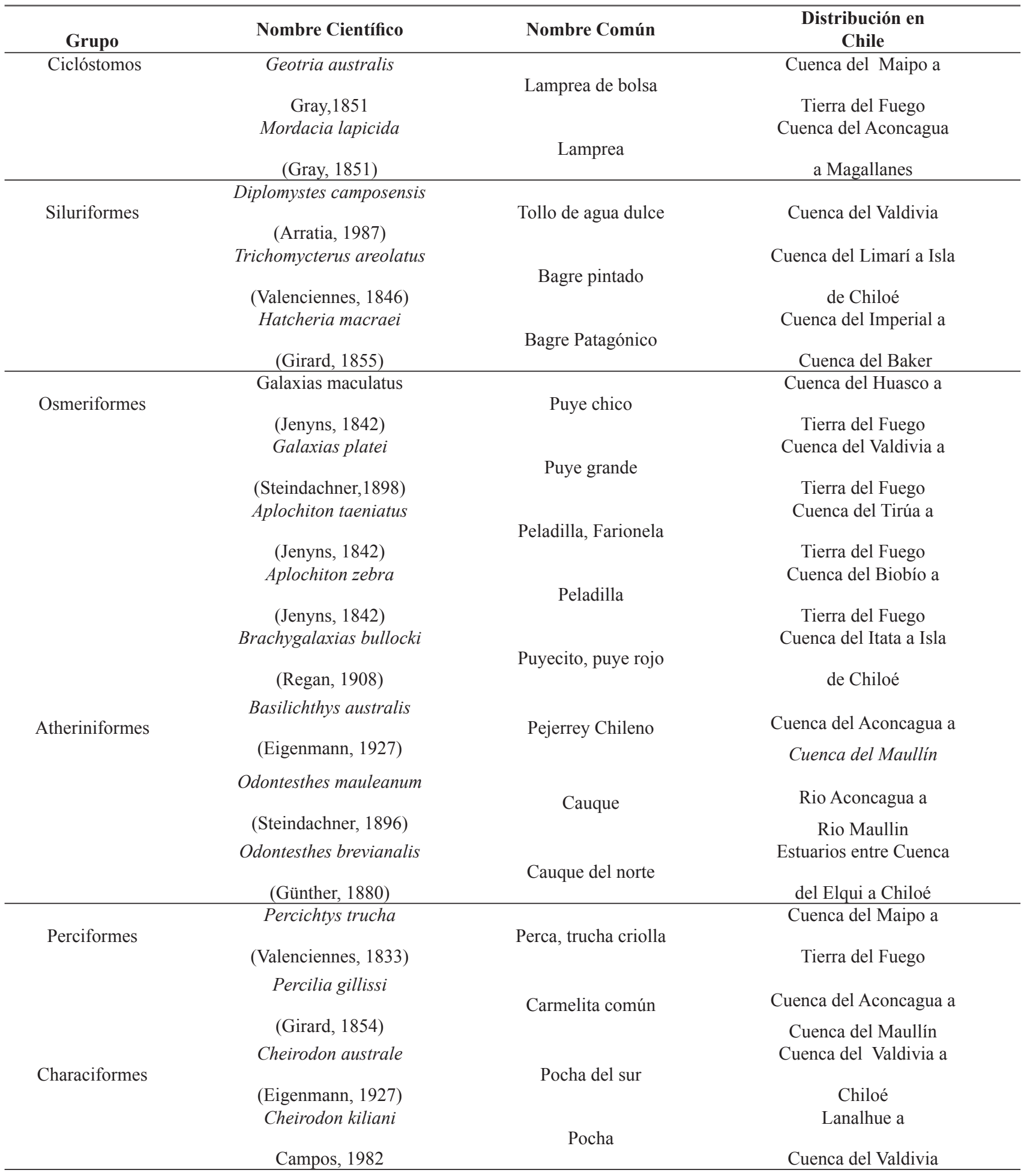


Trichomycterus areolatus (Valenciennes, 1840) ("bagre"; Figura 2c, Tabla 1)

Es una de las especies nativas de más amplia distribución geográfica en Chile, i.e., cuenca del Limarí a la Isla de Chiloé y de alta abundancia en los ríos del país. Por estas características, es tal vez una de las especies más estudiadas en Chile en cuanto a su dieta (Habit 2005; Scott et al. 2007), reproducción (Manríquez et al. 1988), preferencia de hábitats (Arratia 1983; García et al. 2011) y patrones genéticos - filogeográficos (Unmack et al. 2009a; QuezadaRomegialli et al. 2010). Se le encuentra a lo largo de todo el río San Pedro.

Hatcheria macraei (Girard, 1855) ("bagre patagónico"; Figura 2d, Tabla 1)

El hallazgo de $H$. macraei en la cuenca del Valdivia es reciente (Unmack et al. 2009b). Esta especie es poco abundante en el río San Pedro y se encuentra mejor representada en el sector de confluencia con el río Mañío. Se le distingue de $T$. areolatus por la longitud y forma de la aleta dorsal, además del mayor número de rayos en ella.

Galaxias maculatus (Jenyns, 1842) ("puye"; Figura 2e, Tabla 1)

Presenta una distribución gondwánica, estando presente en Nueva Zelanda, Tasmania, Argentina y Chile (Berra et al. 1996; Waters \& Burridge 1999; Cussac et al. 2004). Es una especie diádroma, pero también establece poblaciones residentes o "landlocked" (Campos 1970ab; Peredo \& Sobarzo 1994). Se han registrado diferencias en la morfología y número de vértebras entre poblaciones diádromas y residentes (McDowall 1972, 2003; Campos 1974, Zattara 2002), pero éstas no son completamente indicativas de una condición u otra (Cussac et al. 2004). Recientemente, Zemlak et al. (2010) describen evidencia de estructuración génica entre lagos y ríos, probablemente debida a atributos biológicos como el comportamiento migratorio o residente. Los mismos autores demuestran que las poblaciones de esta especie, provenientes de los lagos de la cuenca del río San Pedro, son filogenéticamente más cercanas a las poblaciones de Argentina (cuencas de los ríos Limay y Manso), que a otras poblaciones cercanas en Chile, como el mismo río Valdivia. Tal patrón sería explicado por la reversión pretérita de cuencas con drenaje Atlántico hacia el Pacífico (Zemlak et al. 2010). En el río San Pedro G. maculatus tiene una distribución continua desde el desagüe del lago Riñihue hasta el río Calle-Calle.

Galaxias platei Steindachner, 1898 ("puye grande"; Figura 2f, Tabla 1)

Esta especie presenta una amplia distribución en Argentina y Chile. Presenta adaptaciones a climas muy rigurosos, así como hábitos bentónicos en aguas lacustres profundas, retina adaptada a ambientes oscuros con aguas de alta carga de sólidos, presencia de una línea lateral cefálica, protección de las branquias contra la abrasión, tolerancia a bajas concentraciones de oxígeno y baja tasa metabólica (Cussac et al. 2004). Tanto las hembras como los machos reproductivos de G. platei habitan en ambientes profundos $(>30 \mathrm{~m})$ de lagos (Barriga et al. 2002). Luego, las larvas migrarían a las zonas limnéticas donde se desarrollan hasta juveniles. Estudios recientes indican que esta especie ha persistido en ambientes glaciados y que han pasado por severos cuellos de botella poblacionales (Ruzzante et al. 2008; Zemlack et al. 2008). En el río San Pedro se la encuentra desde el desagüe del lago Riñihue hasta el río Calle-Calle.

Aplochiton taeniatus Jenyns, 1842, y A. zebra Jenyns, 1842 ("peladillas o farionela"; Figuras $2 \mathrm{~g}$ y $2 \mathrm{~h}$, Tabla 1)

Según Habit et al. (2010), los juveniles de ambas especies de Aplochiton habitan en ambientes someros con rápidos y luego se mueven a lagos o zonas profundas de lagos donde habitan los adultos. Actualmente son raros en los tributarios de lagos pero sí habitan en las zonas litorales lacustres, en la vecindad de la entrada de tributarios a los lagos. Aplochiton taeniatus se distribuye en el río San Pedro, entre el desagüe del lago Riñihue hasta el Balsadero. Aplochiton zebra, en tanto, tiene una distribución más restringida en el río, encontrándose sólo entre el lago Riñihue y el sector de Malihue.

Brachygalaxias bullocki (Regan, 1908) ("puyecito, puye rojo"; Figura 2i, Tabla 1)

Esta es una especie de pequeño tamaño de la cual se cuenta con muy pocos antecedentes biológicos. Es endémica de Chile y se distribuye desde la cuenca del río Itata hasta la Isla de Chiloé. Tal como las especies de Aplochiton, el rango de distribución de $B$. bullocki se ha reducido en la última década principalmente en cuencas con alta intervención humana (Habit et al. 2010). Aún cuando se encontraron unos pocos ejemplares de esta especie en el lago Riñihue, su distribución en el río San Pedro está más restringida a las zonas bajas, en las cercanías de la confluencia del río Quinchilca.

Basilichthys australis Eigenmann, 1927 y Odontesthes mauleanum (Steindachner, 1896) ("Pejerrey chileno" y "Cauque"; Figuras 2j y 2k, Tabla 1)

Corresponden a los pejerreyes nativos de agua dulce más comunes en Chile. Ambas especies se caracterizan porque sus adultos pueden alcanzar tallas grandes en relación a las otras especies nativas ( $>20 \mathrm{~cm}$ longitud total). Ello tiene dos implicancias: por un lado son especies potencialmente valiosas para la pesca deportiva y en segundo lugar, una mayor talla supone una mayor capacidad de desplazamiento. Los antecedentes sobre la biología de estas especies indican que desovan en ambientes de características lénticas (lagos o pozones de ríos), donde emergen los reclutas (Campos et 
al. 1993). Luego, los juveniles usan ambientes ribereños o litorales como hábitat de crianza. Basilichthys australis está distribuida a lo largo de todo el río San Pedro, en tanto que O. mauleanum se la encuentra asociada al lago Riñihue y a la zona inferior del San Pedro.

Percichthys trucha (Valenciennes, 1833) ("Perca trucha"; Figura 21, Tabla 1)

La perca es una de las especies de más amplia distribución en Chile (río Maipo a Tierra del Fuego), que puede alcanzar grandes tallas cuando adulto $(45 \mathrm{~cm}$ longitud total). Se caracteriza por su primera aleta dorsal espinosa y fuertes escamas ctenoídeas ásperas al tacto. Los adultos generalmente se capturan en pozones de río o en lagos, en tanto que los juveniles se asocian a ambientes rocosos ribereños. Esta especie es también muy abundante en la Patagonia argentina (Ruzzante et al. 2006). Se distribuye a lo largo de todo el río San Pedro.

Percilia gillissi Girard, 1854 ("Carmelita"; Figura 2m, Tabla 1)

Existen dos especies de "carmelitas" descritas en Chile, i.e., la "Carmelita de Concepción" o P. irwini (Eigenmann, 1927), descrita para las cuencas del Andalién y Biobío, y $P$. gillissi que se distribuiría desde la cuenca del río Aconcagua a la del río Maullín. Análisis genéticos en el rango distribucional completo indican que existirían dos especies, pero con distribuciones diferentes: una especie del Aconcagua al Biobío y otra especie en el rango sur (Ruzzante et al. 2006). En tanto no se resuelva el problema taxonómico, aquí seguimos utilizando la nomenclatura clásica, en que la especie presente en la cuenca del Valdivia corresponde a $P$. gillissi. Su distribución en el río San Pedro abarca todo su recorrido.

Cheirodon australe Eigenmann, 1927 ("Pocha"; Figura 2n, Tabla 1)

El grupo de las "pochas" es de gran interés por su interesante distribución en Chile, con tres especies alopátricas en sentido latitudinal (Ch. pisciculus Girard, 1855, Ch. galusdae Eigenmann, 1927 y Ch. australe Eigenmann, 1927), además de una especie rara (Ch. kiliani). Son especies abundantes en ambientes lénticos con abundantes macrófitas, así como las zonas bajas de los ríos o potamón. En el río San Pedro se la encuentra desde el desagüe del lago Riñihue hasta el Calle-Calle.

Especies Í́cticas Introducidas y actividades de Pesca en el Río SAn Pedro

Las especies introducidas en la cuenca del río Valdivia corresponden a las truchas café (Salmo trutta (Linnaeus, 1758) y arcoiris (Oncorhynchus mykiss (Walbaum, 1792), ambas presentes en el río San Pedro (Figuras 2o, 2p; Tabla 2), a la carpa común (Cyprinus carpio (Linnaeus, 1758) y al pez mosquito (Gambusia holbrooki (Girard, 1859). Estas dos últimas especies están presentes y son abundantes en las zonas bajas de la cuenca y particularmente en el río Cruces. En el río San Pedro no han sido registradas, probablemente debido a su excelente calidad del agua, bajas temperaturas y mayor velocidad de corriente. Aún cuando lugareños mencionaron la presencia de carpas en el lago Riñihue no las capturamos en ninguna de las numerosas prospecciones efectuadas en el área. La ausencia de la carpa común y el pez mosquito en el río San Pedro, son también indicadores de su buena calidad ambiental, ya que ambas son especies tolerantes a mala calidad del agua e invasoras de sistemas eutróficos. Por el contrario, la presencia de las truchas es un indicador de buena calidad del agua, ya que éstas son especies muy exigentes de altos niveles de oxigenación del agua, bajas concentraciones de nutrientes y bajas temperaturas. Tanto la trucha café como la arcoiris sustentan la pesca recreativa y furtiva que se realiza en el río San Pedro. En el río San Pedro, la pesca recreativa se desarrolla principalmente asociada al desagüe del Lago Riñihue, donde existen instalaciones turísticas que proveen de boteros para hacer pesca embarcada con mosca o señuelo artificial. Visitantes esporádicos o lugareños hacen pesca de orilla con carnada o señuelo artificial. A lo largo del río propiamente tal, la pesca recreativa no es intensiva dado que el río San Pedro escurre en gran parte encajonado y por ende no es de fácil accesibilidad. En general, sólo los propietarios o habitantes ribereños hacen pesca eventual para consumo local. Sin embargo, a la altura de la Balsa San Pedro la pesca de orilla, vadeando o flotando es frecuente, como también lo es la pesca furtiva (pesca con artes no permitidos y fuera de temporada de pesca). El principal problema de esta pesca es que no es selectiva y por tanto, no sólo se capturan peces de interés como las truchas, sino que también peces nativos que luego son usados como carnadas o sencillamente descartados. En este sentido, fue frecuente encontrar ejemplares de $D$. camposensis muertos en las riberas ya que es una especie que muerde fácilmente los anzuelos, principalmente los que usan carnada viva, y los pescadores no los retornan al río. Sin embargo, el Decreto Supremo No 878, del 27 de Septiembre del 2011 de la Subsecretaría de Pesca establece la "veda extractiva" para 30 especies nativas de Chile, entre las cuales se encuentra D. camposensis.

Dado que las especies salmonídeas son introducidas, no tienen valor de conservación per se, sino un valor de uso o comercial, debido a que sustentan la actividad de pesca recreativa. En un contexto ecológico, las especies de salmonídeos han sido definidas por la Unión Internacional de la Conservación (IUCN, www.iucn.org), como las especies invasoras de agua dulce más ampliamente distribuidas en el planeta, habiéndose calificado a las dos especies presentes en el río San Pedro, entre las 100 "especies invasivas más dañinas del planeta" ("World's Worst Invasive Alien 
Species" sensu IUCN). Esto genera una paradoja a los intereses de conservación, ya que los peces más conocidos y apetecidos por el público (pescadores y habitantes locales), son también los que generan mayores daños al ecosistema y la fauna nativa (Simon \& Townsend 2003; Gajardo \& Laikre 2002; Soto et al. 2006; Arismendi et al. 2009). Por el contrario, las especies de peces nativos son generalmente de pequeño tamaño (con excepción de percas y pejerreyes cuando adultos), poco apetecidos para su consumo y bastante poco visibles por su coloración similar a la de su hábitat. De esta manera, incentivar el cuidado de las especies nativas, así como la erradicación o reducción de las poblaciones de truchas es una labor difícil de llevar a cabo. Desde una perspectiva de conservación, el río San Pedro aún alberga en su gran mayoría a peces nativos y no a truchas, por lo cual es factible pensar en la coexistencia de la actividad de pesca y la conservación de la fauna nativa.
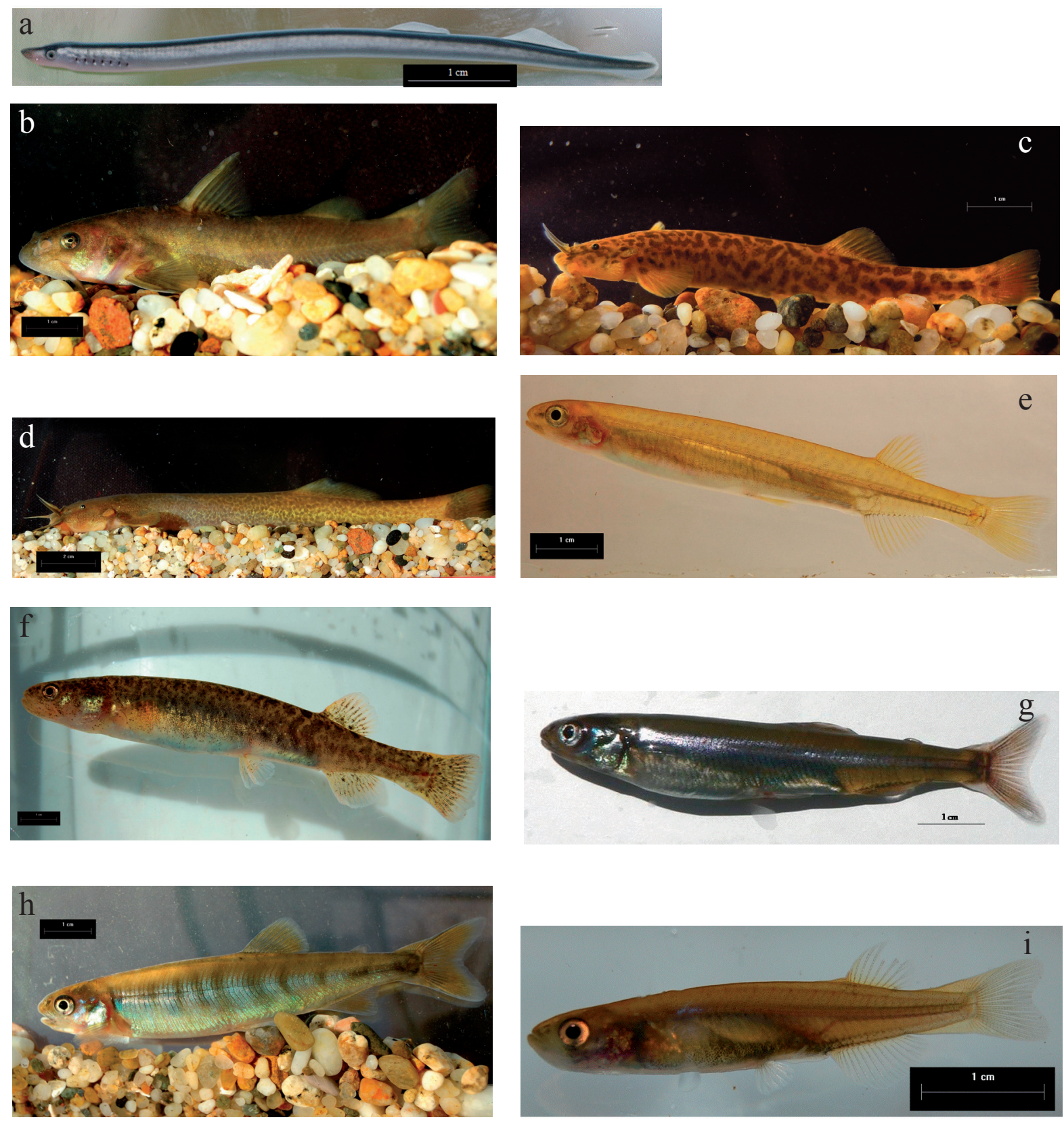

FiguRA 2 
Continuación FIgURA 2
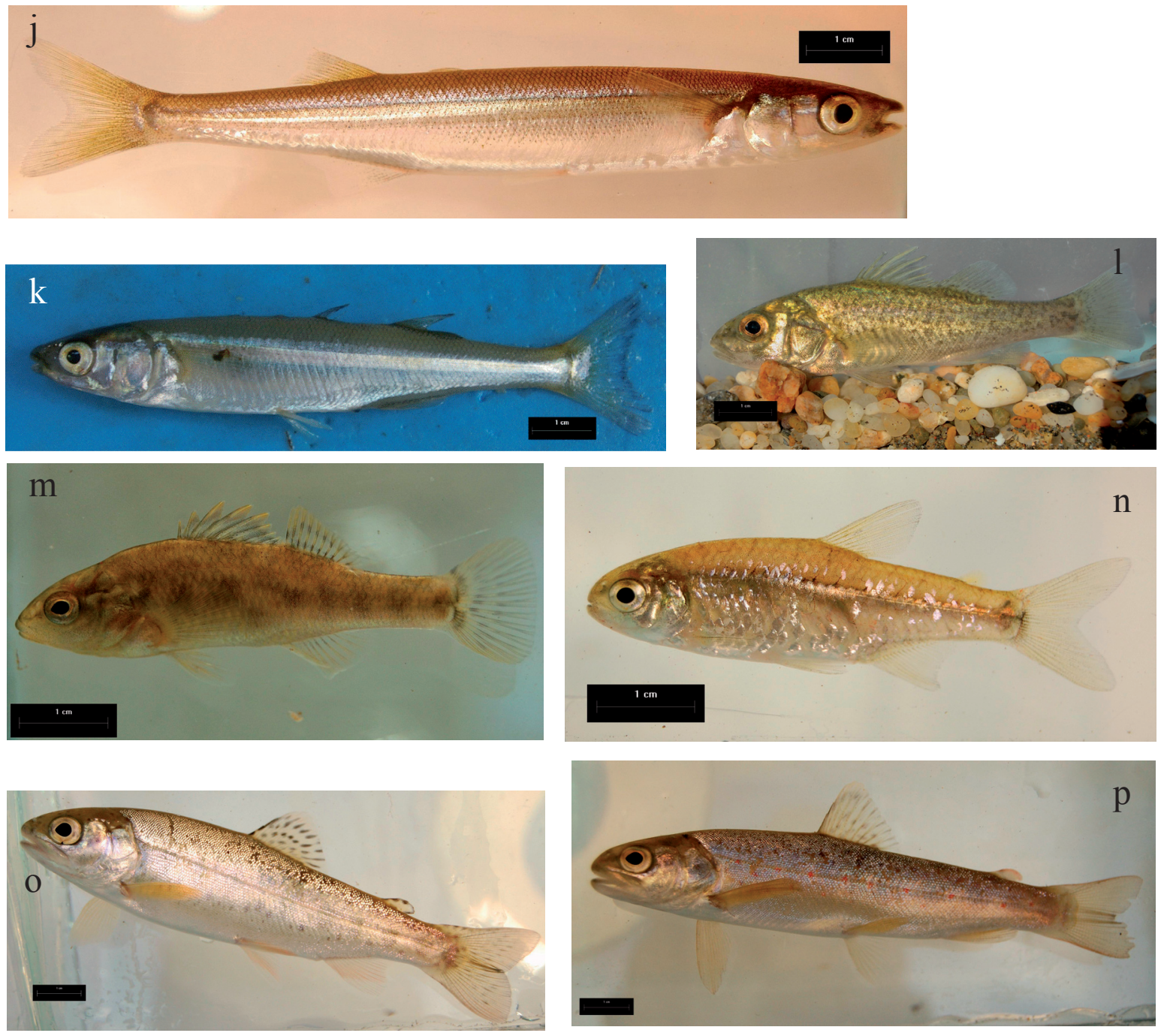

Figura 2. Especies presentes en el río San Pedro. a) Geotria australis (Foto: Richard Mayden); b) Diplomystes camposensis (Foto: Germán Montoya); c) Trichomycterus areolatus (Foto: Germán Montoya); d) Hatcheria macraei (Foto: Germán Montoya); e) Galaxias maculatus (Foto: Germán Montoya); f) Galaxias platei (Foto: Germán Montoya); g) Aplochiton taeniatus; h) Aplochiton zebra (Foto: Germán Montoya); i) Brachygalaxias bullockii (Foto: Gloria Quevedo); j) Basilichthys australis (Foto: Germán Montoya); k) Odontesthes mauleanum (Foto: Roberto Cifuentes); 1) Percichthys trucha (Foto: Germán Montoya); m) Percilia gillissi (Foto: Germán Montoya); n) Cheirodon australe (Foto: Germán Montoya); o) Oncorhynchus mykiss (Foto: Germán Montoya); p) Salmo trutta (Foto: Germán Montoya).

Figure 2. Fish species present in the San Pedro River. a) Geotria australis (Photo: Richard Mayden); b) Diplomystes camposensis (Photo: Germán Montoya); c) Trichomycterus areolatus (Photo: Germán Montoya); d) Hatcheria macraei (Photo: Germán Montoya); e) Galaxias maculatus (Photo: Germán Montoya); f) Galaxias platei (Photo: Germán Montoya); g) Aplochiton taeniatus; h) Aplochiton zebra (Photo: Germán Montoya); i) Brachygalaxias bullockii (Photo: Gloria Quevedo); j) Basilichthys australis (Photo: Germán Montoya); k) Odontesthes mauleanum (Photo: Roberto Cifuentes); 1) Percichthys trucha (Photo: Germán Montoya); m) Percilia gillissi (Photo: Germán Montoya); n) Cheirodon australe (Photo: Germán Montoya); o) Oncorhynchus mykiss (Photo: Germán Montoya); p) Salmo trutta (Photo: Germán Montoya). 
Alcances sobre los orígenes de la ictiofauna del Río San PEDRo

Los ríos chilenos, especialmente aquellos al sur de los $38^{\circ} \mathrm{S}$, han estado sometidos a grandes cambios climáticos cíclicos, los cuales han implicado alternancias de períodos fríos (glaciales), y templados (interglaciales) (Moreno et al. 1994; Hulton et al. 2002). Tales cambios climáticos, junto a la dinámica historia orogenética del relieve chileno, han sido los más importantes como moduladores de la estructuración y procesos de dispersión poblacional. Las oscilaciones de regímenes térmicos habrían generado importantes variaciones en la distribución espacial de las cuencas y en su magnitud espacial, provocando tanto una interesante dinámica de trasvasije de faunas entre las vertientes oriental y occidental de Los Andes, como importantes alteraciones demográficas (Ruzzante et al. 2006, Zemlak et al. 2008, 2010). Entre los efectos más frecuentes de las glaciaciones esta la reducción del rango de distribución espacial, con la consecuente reducción del tamaño efectivo poblacional, y reducciones considerables de la variabilidad genética (Figura 3). Así por ejemplo, cuando tales sistemas han estado sometidos a la presencia de grandes masas de hielo, algunas especies han sido excluidas por completo de áreas que quedaron cubiertas por el hielo, como es el caso de las cuencas hidrográficas al sur de los $42^{\circ} \mathrm{S}$ en la vertiente occidental de Los Andes, provocando la ocurrencia de refugios en áreas aisladas y marginales a los hielos en la vertiente oriental de Los Andes. De este modo, dependiendo de los atributos biológicos de las especies de peces, algunas con altos niveles de tolerancia a la salinidad y anádromas, pudieron refugiarse en ambientes salobres y marinos (e.g. Galaxias maculatus; Zemlak et al. 2011), mientras otras debieron refugiarse en fragmentos no glaciados o en áreas periglaciares (e.g. Galaxias platei; Ruzzante et al. 2008, Zemcak et al 2011). En una magnitud variable, pero siempre reduciendo los tamaños efectivos poblacionales, y por ende, reduciendo los niveles de variabilidad genética, las glaciaciones han sido un proceso fundamental en la modulación de la distribución espacial de las poblaciones y sus niveles de variabilidad genética, y consecuentemente de los potenciales de evolución de las especies afectadas. En este contexto, los cambios climáticos asociados a las glaciaciones, pudieron generar depuración genética $\mathrm{y}$ fragmentación, con la consecuente estructuración de las poblaciones. En síntesis, las glaciaciones habrían generado por una parte, reducciones de biodiversidad genética a nivel local, y por otro probablemente incrementaron la diversificación interpoblacional. Esto último reviste gran importancia pues, en cierto modo las glaciaciones han promovido la divergencia evolutiva entre poblaciones

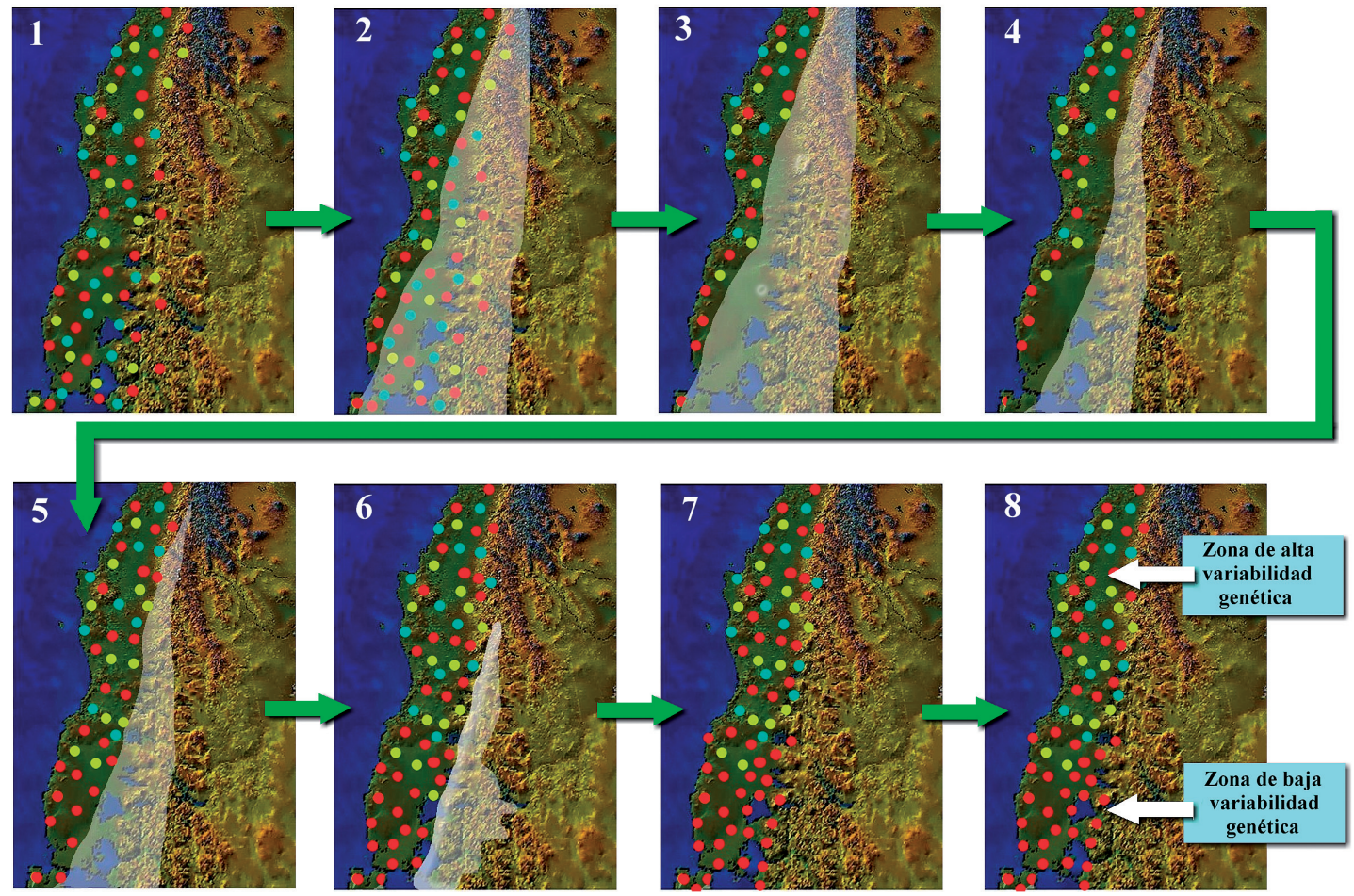

Figura 3. Secuencia temporal hipotética del comportamiento de la distribución geográfica de la variabilidad genética bajo el efecto de las glaciaciones. Los puntos coloreados simbolizan distintos haplotipos, portados por distintos individuos. El área blanca simboliza la cobertura de hielo.

Figure 3. Hypothetic temporal sequence of the geographic distribution of the genetic variability under glaciations effect. Colorful dots represent different haplotypes of different individuals. White area represents ice coverage. 
locales, lo cual constituye un aspecto fundamental al momento de valorar áreas para fines de conservación biológica. En el caso del río San Pedro, éste se encuentra dentro de un área que fue sometida parcialmente a la cobertura glacial, situándose en la zona de la diagonal de hielos que progresivamente se estrechaba hacia el norte (Figura 4). El nacimiento del río San Pedro, situado en la desembocadura del lago Riñihue (Figura 5), se sitúa en un área que fue cubierta por los hielos de las glaciaciones, y por ende corresponde a un área sometida a eventos de recolonización poblacional recurrentes.

Esto significa que, al menos parte de la ictiofauna que actualmente existe en el tramo superior del río, corresponde a poblaciones cuya edad no superaría los 14.000 años. Sin embargo, la magnitud del empobrecimiento genético dependerá de caracteres idiosincráticos o propios de cada especie. Así por ejemplo, algunas poblaciones fuente a partir de las que provinieron los grupos recolonizadores habrían provenido de zonas bajas cercanas a la costa pacífica, mientras otras habrían surgido desde refugios que persistieron en la vertiente oriental de Los Andes. Existe evidencia genética de este último tipo de eventos para peces nativos de Chile, los cuales habrían estado asociados a cambios de la dirección Este a Oeste en que los cursos de agua desembocaban al mar (Ruzzante et al. 2006; Zemlack et al. 2008, 2010). En la parte sur de Los Andes, al sur de los $39^{\circ} \mathrm{S}$, los cordones montañosos son más bajos y más débiles en el sentido de su efecto de barrera biogeográfica, razón por la cual algunos ríos durante la presencia de grandes masas de hielo vertían sus aguas hacia el Atlántico, los cuales, después de cierto umbral de deglaciación, habrían terminado vaciando sus aguas hacia la vertiente Pacífica, y por ende aportando con ictiofauna al territorio chileno, dentro de las cuales está el río San Pedro. En este tipo de escenarios, incluso especies cercanamente relacionadas desde un punto de vista filogenético, como las del género Galaxias, habrían tenido un comportamiento poblacional distinto. Galaxias maculatus, especie diádroma, pudo recolonizar tanto a partir de refugios transandinos, como costero-pacíficos, en cambio G. platei, estrictamente de aguas continentales, habría recolonizado predominantemente desde refugios transandinos (Ruzzante et al. 2008). Este es un ejemplo de lo compleja que puede ser la dinámica histórica de un área en términos de las respuestas demográficas y microevolutivas que han experimentado las especies de peces actualmente co-distribuidas en el río San Pedro.

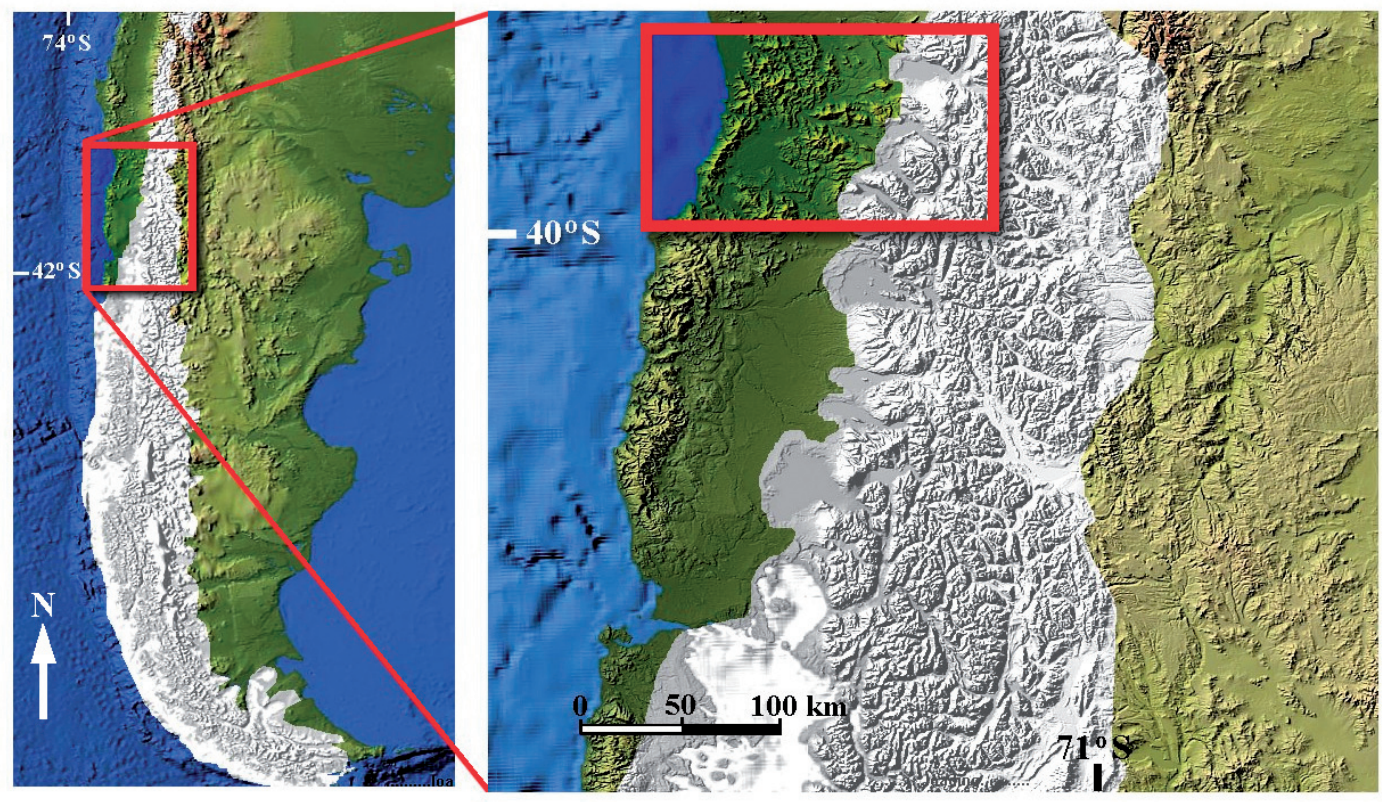

FiguRa 4. Mapa que muestra la distribución aproximada de la capa de hielo durante el Ultimo Máximo Glacial (UMG). El recuadro rojo de la derecha indica el área de estudio. Basado en McCulloch et al. (2005) y Hulton et al. (2002).

FIgURE 4. Ice coverage distribution during the last maximum glacier (LGM). Red square represents the study area. Based on McCulloch et al. (2005) and Hulton et al. (2002). 


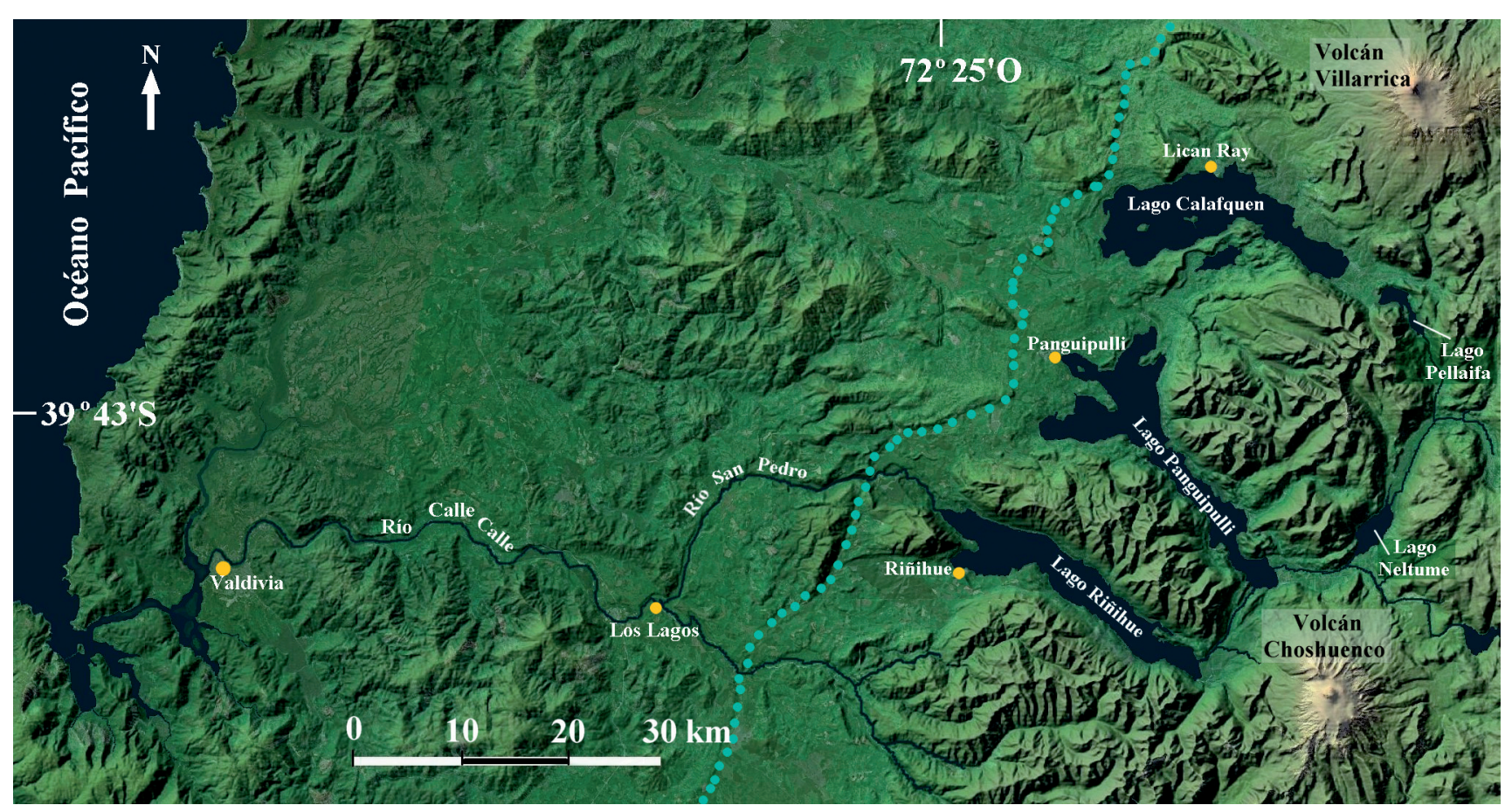

Figura 5. Mapa toponímico del área de estudio. La línea punteada indica el límite aproximado de la placa de hielos durante el Último Máximo Glacial.

Figure 5. Study area map. Dotted line indicates the ice sheet limit during the last máximum glacier.

Estado de Conservación de la Ictiofauna Nativa del río SAN PEDRO

La Tabla 3 muestra los estados de conservación de las 14 especies nativas presentes en el río San Pedro, según diferentes clasificaciones. Las dos primeras, corresponden a las clasificaciones nacionales más ampliamente utilizadas hasta el presente, las cuales están basadas en la opinión de expertos más que en datos cuantitativos, como son el Libro Rojo de los Vertebrados Terrestres de Chile (Glade 1988), y Boletín del Museo Nacional de Historia Natural (Campos et al. 1998). Ambas clasificaciones presentan para cada especie un estado de conservación a nivel nacional (país) y a nivel regional (regiones administrativas), y muestran bastantes coincidencias entre ellas. Según éstas, en el río San Pedro existirían tres especies en Peligro de Extinción, correspondientes a Diplomystes camposensis (aún cuando Campos et al. 1998 la declara Vulnerable), Aplochiton taeniatus y Aplochiton zebra (aún cuando Glade, 1988 define esta última como Vulnerable). La última clasificación efectuada en Chile se ha llevado a cabo bajo el Reglamento de
Clasificación de Especies Silvestres de CONAMA (Decreto No75 de 2004) y publicado como Decreto 51 del 2008. Esta clasificación deja varias de las especies sin Propuesta de Clasificación o Fuera de Peligro, en cuencas hidrográficas del Biobío hacia el sur. CONAMA (2008), al igual que en el Libro Rojo (Glade 1988), considera a D. camposensis en Peligro de Extinción. La clasificación internacional de IUCN es también incompleta, ya que se basa en datos cuantitativos exigentes, de los cuales se carece de información para la mayoría de las especies nativas chilenas. Por ello, el resultado es que gran parte de las especies presentes en el río San Pedro no están clasificadas o se las clasifica como "Data deficient" o falta de información para su clasificación (Tabla 3).

Finalmente concluimos que la ictiofauna del río San Pedro es de alto valor de conservación, así como de alta riqueza específica, comparativamente con otros sistemas fluviales de Chile. 
TABLA 3. Estado de conservación de los peces presentes en el río San Pedro según distintas clasificaciones nacionales (Libro Rojo, Boletín 47 MNHN y CONAMA) e internacionales (IUCN, Red List). Cuando corresponde, se indica la categoría a nivel de país y entre paréntesis en la X Región (actualmente XIV Región) donde se ubica la cuenca del Valdivia. V: Vulnerable; FP: Fuera de Peligro; P: En Peligro; I: Indeterminado; IC: Insuficientemente Conocida; SPC: Sin Propuesta de Clasificación; NC: No Clasificada; DI: Datos Insuficientes; DD: "Data Deficient" (datos insuficientes); LR/nt: "Low Risk/near threatment" (bajo riesgo / cerca de amenazada).

TABLE 3. Conservation status of the fish species in the San Pedro River, based on different national (Libro Rojo, Boletín 47 MNHN and CONAMA) and international classifications (IUCN, Red List). When apply, we included regional conservation status for the X Region (currently XIV Region), from where the Valdivia River belongs. V: Vulnerable; FP: No Risk; P: Endangered; I: Indeterminate; IC: Data deficient; SPC: Not classified; NC: Not classified; DI: Data deficient; DD: Data Deficient; LR/nt: Low Risk/near threatment.

\begin{tabular}{|c|c|c|c|c|}
\hline \multirow{2}{*}{ Nombre Científico } & \multicolumn{3}{|c|}{ Clasificaciones Nacionales } & \multirow{2}{*}{$\begin{array}{c}\begin{array}{c}\text { Clasificación } \\
\text { Internacional }\end{array} \\
\text { IUCN } \\
\text { (Red List 2007) }\end{array}$} \\
\hline & $\begin{array}{l}\text { Libro Rojo } \\
\text { (1989) }\end{array}$ & $\begin{array}{c}\text { Bol. } 47 \mathrm{MNHN} \\
\text { (1998) }\end{array}$ & $\begin{array}{l}\text { CONAMA } \\
(2008)\end{array}$ & \\
\hline Geotria australis & $\mathrm{V}(\mathrm{V})$ & $\mathrm{V}(\mathrm{V})$ & FP & $\mathrm{NC}$ \\
\hline Diplomystes camposensis & $\mathrm{P}(\mathrm{P})$ & $\mathrm{V}(\mathrm{V})$ & $\mathrm{P}$ & DD \\
\hline Trichomycterus areolatus & $\mathrm{V}(\mathrm{FP})$ & $\mathrm{V}(\mathrm{FP})$ & $\mathrm{V}$ & DD \\
\hline Hatcheria macraei & Rara & Rara & IC Rara & $\mathrm{NC}$ \\
\hline Galaxias maculatus & $\mathrm{V}(\mathrm{V})$ & $\mathrm{V}(\mathrm{FP})$ & FP & $\mathrm{NC}$ \\
\hline Galaxias platei & $\mathrm{V}(\mathrm{V})$ & $\mathrm{V}(\mathrm{V})$ & FP & $\mathrm{NC}$ \\
\hline Aplochiton taeniatus & $\mathrm{P}(\mathrm{P})$ & $\mathrm{P}(\mathrm{P})$ & $\mathrm{SPC}$ & $\mathrm{NC}$ \\
\hline Aplochiton zebra & $\mathrm{V}(\mathrm{V})$ & $\mathrm{V}(\mathrm{P})$ & SPC & $\mathrm{NC}$ \\
\hline Brachygalaxias bullocki & $\mathrm{V}(\mathrm{V})$ & $\mathrm{I}(\mathrm{I})$ & $\mathrm{FP}$ & $\mathrm{NC}$ \\
\hline Basilichthys australis & $\mathrm{V}(\mathrm{V})$ & $\mathrm{V}(\mathrm{FP})$ & FP & $\mathrm{LR} / \mathrm{nt}$ \\
\hline Odontesthes mauleanum & $\mathrm{V}(\mathrm{V})$ & $\mathrm{V}(\mathrm{V})$ & $\mathrm{V}$ & $\mathrm{LR} / \mathrm{nt}$ \\
\hline Percichtys trucha & $\mathrm{V}(\mathrm{V})$ & $\mathrm{V}(\mathrm{V})$ & FP & $\mathrm{NC}$ \\
\hline Percilia gillissi & $\mathrm{V}(\mathrm{V})$ & $\mathrm{V}(\mathrm{V})$ & $\mathrm{SPC}$ & DD \\
\hline Cheirodon australe & $\mathrm{V}(\mathrm{V})$ & $\mathrm{FP}(\mathrm{FP})$ & $\mathrm{V}$ & DD \\
\hline
\end{tabular}




\section{AGRADECIMIENTOS}

Agradecemos a la Empresa COLBÚN S.A. por financiar este estudio y al proyecto DIUC Semilla Patagonia 210.310.0571SP por el financiamiento de la publicación. A Catalina Puga del Hotel Riñimapu y propietarios de predios aledaños al río San Pedro, por su importante colaboración en la realización del trabajo de campo a lo largo de todos los años de este estudio. Igualmente se agradece a todas las personas que directa o indirectamente participaron en la elaboración de este estudio. Finalmente se agradece al proyecto Fondecyt 1110441 que permitió la finalización de este manuscrito.

\section{BIBLIOGRAFIA}

Arismendi, I., D. Soto, B. Penaluna, C. Jara, C. Leal \& León-Muñoz, J. 2009. Aquaculture, non-native salmonid invasions and associated declines of native fishes in northern Patagonian lakes. Freshwater Biology 54:1135-1147.

Arratia, G. 1983. Preferencias de hábitat de peces siluriformes de aguas continentales de Chile (Fam. Diplomystidae y Trichomyctidae). Studies of Neotropical Fauna and Environment 18(4): 217-237.

Arratia, G. 1987. Pisces: Diplomystidae. Gayana Oceanología 4(2): 129-137.

Arratia, G. 1992. Development and variation of the suspensorium of primitive catfishes (Teleostei: Ostariophysi) and their phylogenetic relationships. Bonner zoologische Monographien 32: 1-149.

Barriga, J., Milano, D., Batín, M., Macchi, M. \& Cussac, V. 2002. Spatial and temporal distribution of landlocked Galaxias maculatus and Galaxias platei (Pisces, Galaxiidae) in a lake in South American Andes. New Zealand Journal of Marine and Freshwater Research 36: 349-363.

Benn, I.D. \& Evans, D.J. 2010. Glaciers and Glaciation. Oxford University Press, USA. 816 pp.

Berra, T., Mcrowley, L., Ivantsoff, W. \& Fuerst, P. 1996. Galaxias maculatus: an explanation of its biogeography. Marine and Freshwater Research 47: 845-849.

CAmpos, H. 1970a. Introducción de especies exóticas y su relación con los peces de agua dulce de Chile. Noticiaro Mensual Museo Nacional Historia Natural, Chile, 14(162): 3-9.

CAmpos, H. 1970b. Galaxias maculatus (Jenyns) en Chile, con especial referencia a su reproducción. Boletin Museo Nacional Historia Natural, Chile. 31: 5-20.

CAmpos, H. 1974. Population studies of Galaxias maculatus (Jenyns) (Osteichthyes: Galaxiidae) in Chile with reference to the number of vertebrae. Studies on Neotropical Fauna and Environment 9: 55-76.

CAmpos, H. 1985. Distribution of the fishes in the Andean rivers in the South of Chile. Archives Hydrobiology 104(2): 69 $-191$.

Campos, H., Gavilán, J., Alay, F. \& Ruiz, V. 1993. Comunidad íctica de la hoya hidrográfica del Río Biobío. Gestión de recursos hídricos de la cuenca del Río Biobío y del área marina costera adyacente. F FARANDA and O PARRA. Concepción, Chile, Centro EULA-Chile, Universidad de Concepción. Serie: Monografías científicas Vol XII: 249-
278.

Campos, H., Dazarola, G., Dyer, B., Fuentes, L., Gavilán, J., Huaquín, L., Martínez, G., Meléndez, R., Pequeño, G., Ponce, F., Ruiz, V., Siefeld, W., Soto, D., Vega, R. \& Vila, I. 1998. Categorías de conservación de peces nativos de aguas continentales de Chile. Boletín del Museo Nacional de Historia Natural, Chile 47: 101-122.

Conama. 2008. Aprueba y Oficializa Nómina para el Tercer Proceso de Clasificación de Especies Según su Estado de Conservación. DS 51 del 24 de abril 2008.

Cussac, V., Ortubay, S., Iglesias, G., Milano, D., Lattuca, E., Barriga, P., Battini, M. \& Gross, M. 2004. The distribution of South American galaxiid fishes: the role of biological traits and post-Glacial history. Journal of Biogeography 31: 103-121.

De Pinna, M. 1998. Phylogenetic relationships of neotropical siluriformes (Teleostei: Ostariophysi): historical overview and synthesis of hypotheses. Phylogeny and Classification of Neotropical Fishes, L.R. Malabarba, R.E. Reis, R.P. Vari, Z.M. Lucena \& C.A. Lucena, Edipucrs, Porto Alegre, Brasil. 279-330 pp.

Dyer, B. 2000. Systematic review and biogeography of the freshwater fishes of Chile. Estudios Oceanológicos 19: 77 - 98 pp.

Gajardo, G. \& Laikre, L. 2002. Chilean aquaculture boom is based on exotic Salmon resources: a conservation paradox. Conservation Biology 17(4): 1173-1174.

García, A., Jorde, K., Habit, E., CaAmaño, D., \& Parra, O. 2011. Downstream environmental effects of dam operations: Changes in habitat quality for native fish species. River Research and Applications 27: 312 -327.

Glade, A. 1988. Libro rojo de los vertebrados terrestres de Chile. Actas del Simposio: Estado de Conservación de los Vertebrados Terrestres de Chile. CONAF, Santiago. 67 pp.

GRANDE, L. 1987. Redescription of †Hypsidoris farsonensis (Teleostei: Siluriformes), with a reassessment of its phylogenetic relationships. Journal of Vertebrate Paleontology 7: 24-54

Habit, E. 2005. Aspectos de la biología y hábitat de un pez endémico de Chile en peligro de extinción (Diplomystes nahuelbutaensis Arratia, 1987). Interciencia 30: 8-11.

Habit, E., Dyer, B., \& Vila, I. 2006a Estado de conocimiento de los peces dulceacuícolas de Chile: estado de su conocimiento. Gayana 70(1): 100-112.

Habit, E., Belk, M., \& Parra, O. 2006b Response of the fish community to human-induced changes in of the Biobío River in Chile. Freshwater Biology 51:1-11.

Habit, E., Piedra, P., Ruzzante, D., Walde, S., Belk, M., Cussac, V., GonzÁlez, J., \& Colin, N. 2010. Changes in the distribution of native fishes in response to introduced species and other anthropogenic effects. Global Ecology \& Biogeography 19: 697-710.

Hulton, N., Purves, R., McCulloch R., Sugden, D. \& Bentley, M. 2002. The last glacial maximum and glaciation in southern South America. Quaternary Science Reviews 21: 233-241.

Jellyman, D. \& Glova, J. 2002. Habitat use by juvenile lampreys (Geotria australis) in a large New Zealand river. New Zealand Journal of Marine and Freshwater Research. 36: 503-510.

Jellyman, D., Glova, J. \& Sykes, J.R. 2002. Movements and 
habitat of adult lamprey (Geotria australis) in two New Zealand waterways. New Zealand Journal of Marine and Freshwater Research 36: 53-65.

Lundberg, G., Berra, T., Tim, M., Friel, J. \& John, P. 2004 First description of small juveniles of the primitive catfish Diplomystes (Siluriformes: Diplomystidae). Ichthyological Exploration of Freshwaters 15 (1): 71-82.

Manríquez, A., Huaquín, L., Arellano, M. \& Arratia, G. 1988. Aspectos reproductivos de Trichomycterus areolatus Valenciennes, 1846 (Pisces: Teleostei: Siluriformes) en río Angostura, Chile. Studies on Neotropical Fauna and Environment 23: 89-102.

Markgraf, V. 1989. Palaeoclimates in Central and South America since 18,000 BP based on pollen and lake-level records. Quaternary Science Reviews 8: 1-24.

McCulloch, R.D., M. Bentley, R.M. Tipping \& C.M. Clapperton. 2005. Evidence for late-glacial iced dammed lakes in the central strait of Magellan and Bahia Inútil, southernmost South America. Geografiska annaler 87A: 335-362.

McDowall, R. 1972. The species problem in freshwater fishes and the taxonomy of diadromous and lacustrine populations of Galaxias maculatus (Jenyns). Journal of the Royal Society of NewZealand, 2: 325-367.

McDowall, R. 2003. Variation in vertebral number in galaxiid fishes (Teleostei: Galaxiidae): a legacy of life history, latitude and length. Environmental Biology of Fishes 66: 361-381.

Moreno, P., Villagrán, C., Marquet, P. \& Marshall, L. 1994. Quaternary paleobiogeography of northern and central Chile. Revista Chilena de Historia Natural 67: 487-502.

MYers, N. 2003. Biodiversity hotspots revisited. BioScience 53: 916-917.

Peredo, S. \& Sobarzo, C. 1994. Actividad gonádica estacional de Galaxias maculatus (Jenyns, 1842) en el río Cautín, IX Región, Chile. Boletín de la Sociedad de Biología de Concepción, Chile 65: 65-70.

Quezada-Romegialli, C., M. Fuentes \& Véliz, D. 2010. Comparative population genetics of Basilichthys microlepidotus (Atheriniformes: Atherinopsidae) and Trichomycterus areolatus (Siluriformes: Trichomycteridae) in north central Chile. Environmental Biology of Fishes 89:173-186.

Ruiz, V. \& Marchant, M. 2004. Ictiofauna de aguas continentales de Chile. Facultad de Ciencias Naturales y Oceanográficas, Universidad de Concepción. 356 pp.

Ruiz, V. 1993. Ictiofauna del río Andalién (Concepción, Chile). Gayana Zoología 57: 109-278.

Ruzzante, D., Walde, S., Cussac, V., Dalebout, M., Seibert, J., Ortubay, S. \& Habit, E. 2006 Phylogeography of the Percichthyidae (Pisces) in Patagonia: roles of orogeny, glaciation, and volcanism. Molecular Ecology 15(10): 2949-2968.

Ruzzante, D., Walde, S., Gosse, J., Cussac, V., Habit, E., \& Zemlak, T. 2008. Adams EDM: Climate control on ancestral population dynamics: insight from Patagonian fish phylogeography. Molecular Ecology 17:2234-2244.

Scott, S., PARDo, R. \& VILA, I. 2007 Trophic niche overlap between two Chilean endemic species of Trichomycterus (Teleostei:
Siluriformes) Revista Chilena de Historia Natural 80: 431437.

Simon, K. \& Townsend, C. 2003 Impacts of freshwater invaders at different levels of ecological organisation, with emphasis on salmonids and ecosystem consequences. Freshwater Biology 48: 982-994.

Soto, D., I. Arismendi, J. GonzÁlez, J. SAnzana, F. Jara, C. Jara, E. GuZmán \& LARA, A. 2006. Sur de Chile, país de truchas y salmones: patrones de invasión y amenazas para las especies nativas. Revista Chilena de Historia Natural 79: 97-117.

Sullivan, J., Lundberg, G. \& Hardman, M. 2006. A phylogenetic analysis of the major groups of catfishes (Teleostei: Siluriformes) using rag1 and rag2 nuclear gene sequences. Molecular Phylogenetics and Evolution 41: 636-662.

Unmack, P., Bennin, A., Habit, E., Victoriano, P. \& Johnson, J. 2009a. Impact of ocean barriers, topography and glaciation on phylogeography of the catfish Trichomycterus areolatus (Teleostei: Trichomycteridae) in Chile. Biological Journal of the Linnean Society 97: 876-892.

Unmack, P., Habit, E. \& Johnson, J. 2009b. New records of Hatcheria macraei from Chilean Province. Gayana 73(1): 102-110

Valdovinos, C. 2006. Biodiversidad dulceacuícola de Chile. En: Gayana, International Journal of Biodiversity, Oceanology and Conservation. Universidad de Concepción, Chile. 70(1): 1-162.

Vila, I., Fuentes, L. \& Contreras, M. 1999. Peces límnicos de Chile. Boletín del Museo Nacional de Historia Natural, Chile 48: 61-75.

Villagrán, C. \& Armesto, J. 2005. Fitogeografia histórica de la Cordillera de la Costa de Chile. En: Historia. Biodiversidad y Ecología de los bosques costeros de Chile. Smith-Ramirez C., Armesto J., Valdovinos, C. Editorial Universitaria, Santiago, Chile. 99-116 pp.

Waters, J. \& Burridge, C. 1999. Extreme intraspecific mitochondrial DNA sequence divergence in Galaxias maculatus (Osteichthys: Galaxiidae), one of the world's most widespread freshwater fish. Molecular Phylogeny and Evolution 11(1): 1-12.

ZatTARA, E. 2002 Variación isoenzimática y morfológica en poblaciones de Galaxias maculatus Jenyns de los Lagos Andino-Patagónicos. Trabajo final presentado para optar al grado de Licenciado en Ciencias Biológicas. Centro Regional Universitario Bariloche. Universidad Nacional del Comahue. 110 pp.

Zemlak, T., Habit, E., Walde, S., Battini, M., Adams, E. \& Ruzzante, D. 2008. Across the southern Andes on fin: glacial refugia, drainage reversals and a secondary contact zone revealed by the phylogeographical signal of Galaxias platei in Patagonia. Molecular Ecology 17:5049-5061

Zemlak, T., Habit, E., Walde, S., Carrea, C. \& Ruzzante, D. 2010. Surviving historical Patagonian landscapes and climate: molecular insights from Galaxias maculatus. BMC Evolutionary Biology 10:67.

Zemlak, T., Walde, S., Habit, E. \& Ruzzante, D. 2011. Climateinduced changes to the ancestral population size of two patagonian galaxiids: the influence of glacial cycling. Molecular ecology 20: 5280 - 5294.

Recibido: 02.01.12

Aceptado: 27.02.12 